Meta

Journal des traducteurs

Translators' Journal

\title{
Les interprètes japonais en Algérie
}

\section{Michihiro Nohara}

Volume 33, numéro 1, mars 1988

Traduction et interprétation au Japon

Translation and Interpretation in Japan

URI : https://id.erudit.org/iderudit/003743ar

DOI : https://doi.org/10.7202/003743ar

Aller au sommaire du numéro

\section{Éditeur(s)}

Les Presses de l'Université de Montréal

\section{ISSN}

0026-0452 (imprimé)

1492-1421 (numérique)

Découvrir la revue

\section{Citer cet article}

Nohara, M. (1988). Les interprètes japonais en Algérie. Meta, 33(1), 90-93.

https://doi.org/10.7202/003743ar

\section{Résumé de l'article}

Since the end of the sixties, numerous Japanese contractors have been active in heavy industry work in Algeria and other North African countries. This has opened a large market for Japanese-French liaison interpreters. Such interpreters are hired by specialized agencies in Europe and Japan. With one or two exceptions, they are Japanese nationals. Most of them are young and have no previous experience as interpreters. Neither are they properly briefed before they are sent to the site, but they become proficient in their work with experience. Their tasks range from interpreting at various levels to translation and even participation in negotiations and report drafting. Material working conditions are comparatively good, but the duration of contracts is short and long-term stability of employment is uncertain. The precariousness of interpreting jobs and the temporary nature of the Japanese companies' contracting work in North Africa make this activity a provisional and highly volatile one. Japanese liaison interpreters in North Africa do not feel like professional interpreters and do not aspire to become professionals.
Ce document est protégé par la loi sur le droit d'auteur. L'utilisation des services d'Érudit (y compris la reproduction) est assujettie à sa politique d'utilisation que vous pouvez consulter en ligne.

https://apropos.erudit.org/fr/usagers/politique-dutilisation/ 


\title{
LES INTERPRÈTES JAPONAIS EN ALGÉRIE
}

\author{
MICHIHIRO NOHARA, Japon
}

\section{SUMMARY}

Since the end of the sixties, numerous Japanese contractors have been active in heavy industry work in Algeria and other North African countries. This has opened a large market for Japanese-French liaison interpreters. Such interpreters are hired by specialized agencies in Europe and Japan. With one or two exceptions, they are Japanese nationals. Most of them are young and have no previous experience as interpreters. Neither are they properly briefed before they are sent to the site, but they become proficient in their work with experience. Their tasks range from interpreting at various levels to translation and even participation in negotiations and report drafting. Material working conditions are comparatively good, but the duration of contracts is short and long-term stability of employment is uncertain. The precariousness of interpreting jobs and the temporary nature of the Japanese companies' contracting work in North Africa make this activity a provisional and highly volatile one. Japanese liaison interpreters in North Africa do not feel like professional interpreters and do not aspire to become professionals.

Depuis la fin des années soixante, des entreprises japonaises participent à des projets d'industrialisation des pays d'Afrique du Nord, et notamment de l'Algérie où elles ont conclu des contrats se chiffrant à plusieurs milliards de yens dans les domaines de la pétrochimie, la sidérurgie, la métallurgie, les barrages, les centrales hydrauliques, etc., puis dans les industries légères.

L'un des problèmes qui se posent pour les Japonais dans l'exportation de leur savoir-faire dans cette région du monde est d'ordre linguistique : le français, langue véhiculaire en Afrique du Nord, est connu de très peu d'ingénieurs et de techniciens nippons (le problème est sensiblement moins aigu pour les autres pays coopérants, qui arrivent à trouver des ingénieurs et techniciens parlant français). C'est pourquoi initialement, les entreprises japonaises qui démarraient leurs activités en Algérie affectaient des interprètes français-japonais à leurs ingénieurs et techniciens dans la proportion de un à dix environ. Dans des programmes de formation de techniciens et d'ouvriers algériens, la proportion était ramenée de un à cinq. Ces chiffres ne représentent toutefois qu'une moyenne, et il arrive que dans des programmes de formation, il y ait autant d'interprètes que d'expatriés ingénieurs. Le système s'étant consolidé et répandu, l'on assista à la naissance d'un marché de l'interprétation japonais-français. On peut estimer son importance par rapport à l'ensemble du marché de la traduction franco-japonais à $50 \%$ environ pour la traduction et $80 \%$ pour l'interprétation.

À la différence des interprètes de conférence et des interprètes de liaison de type traditionnel, les interprètes japonais en Afrique du Nord (les interprètes franco-japonais n'ayant pas la nationalité japonaise sont extrêmement rares) ont une gamme d'activités très large et interviennent à tous les niveaux et dans tous les aspects du projet auquel ils sont affectés : liaison entre ingénieurs ou techniciens japonais et ouvriers algériens, interprétation à des négociations de haut niveau, intervention dans les rapports avec les autorités algériennes, voire contacts individuels et personnels entre les expatriés japo- 
nais et les ouvriers autochtones. Leur mission principale consiste toutefois à assister les ingénieurs et techniciens japonais dans leur travail quotidien, et ils interviennent surtout dans les séances de travail nippo-algériennes. Selon les circonstances, ils travaillent en consécutive phrase-par-phrase, en "vraie consécutive » ou en chuchotée, mais pas en simultanée.

Par ailleurs, ces interprètes servent également de traducteurs : ils traduisent des dossiers, des rapports, des documents techniques et des manuels d'instruction, et il arrive même qu'ils participent à la rédaction de rapports officiels.

Hormis les très grands projets dans lesquels sont engagés 3000 expatriés japonais et: une centaine d'interprètes, où ceux-ci peuvent être affectés à des services ou fonctions particulières, les interprètes sont dans l'ensemble "polyvalents".

Compte tenu de la variété de leurs tâches, les aptitudes et compétences qui leur sont demandées ne sont pas définies de manière uniforme. Âgés de 20 à 40 ans environ lat moyenne se situant autour de 35 ans - ils sont diplômés d'université ou au moins bacheliers, et sortent soit d'universités ou d'écoles privées japonaises où ils ont appris le français ( $30 \%$ environ), soit d'écoles de français en France ( $70 \%$ environ) ou dans d'autres pays francophones, ce dernier cas étant plus rare. La plupart ont démarré entre 26 et. 28 ans d'âge, entre la fin des années 60 et le début des années 80 , où la demande a été la plus forte. Beaucoup d'entre eux exerçaient un autre métier avant de devenir interprètes, mais rares sont ceux qui avaient une expérience de l'interprétation avant d'être recrutés pour leur travail en Algérie. Actuellement, les interprètes travaillant en Afrique du Nord ont en moyenne 5 à 10 ans d'expérience de l'interprétation.

Recrutés par des agences spécialisées (une vingtaine) au Japon ou à Paris où elles ont des bureaux de liaison, et parfois par des agences de "communication ", ils signent des contrats à durée déterminée pour des missions d'une semaine à deux ou trois ans; ils sont alors considérés comme faisant partie du personnel de ces agences et sont détachés chez les clients.

Leur recrutement se fait en deux étapes : inscription auprès d'une agence et recrutement par l'entreprise. Au moment de l'inscription, ils sont sélectionnés par entretien et tests de traduction et d'interprétation japonais-français dans les deux sens ou conversation en français. La sélection par l'entreprise-client se fait de manière analogue, les interprètes faisant état d'une expérience importante étant parfois dispensés des tests.

Les interprètes traversent une période d'essai qui peut être de trois mois par exemple, mais il est rare qu'ils soient renvoyés une fois arrivés sur place, ce qui serait contraire aux traditions japonaises. Il est vrai aussi que les Japonais sont relativement peu exigeants sur le plan de la qualité du travail. Les Algériens le sont davantage, et sont par ailleurs très sensibles au comportement des interprètes dans leurs relations avec les autochtones.

Hormis de brèves explications générales ou de rares documents techniques qui leur sont remis avant leur départ, les interprètes ne bénéficient pas d'une formation préalable et doivent acquérir "sur le tas » les connaissances spécialisées nécessaires à leur travail, ce que la durée de leur contrat leur permet de faire.

Une fois arrivés sur place, ils sont considérés comme faisant partie de l'entrepriseclient et ont en général un statut de cadres. Une assurance maladie-accidents leur est proposée par leur agence, et ils peuvent la compléter par des assurances additionnelles (chômage, retraite, manque à gagner), et notamment la Sécurité sociale algérienne. Ils bénéficient également, au même titre que les salariés expatriés, de l'assistance médicale des médecins japonais travaillant sur place pour l'entreprise concernée ou venant faire des visites périodiques, et ce au même titre que les salariés de cette entreprise. 
Conformément à la législation du travail algérienne, les interprètes travaillent 44 heures par semaine, avec un jour et demi de congé hebdomadaire en dehors des jours fériés algériens. Les heures supplémentaires sont rémunérées ou compensées par des congés groupés en jours. La durée du congé annuel dépend du contrat signé avec l'agence. A titre d'illustration, pour un contrat de plus d'un an, il est d'une vingtaine de jours. De nombreuses entreprises offrent aux interprètes un billet d'avion aller-retour entre le lieu de travail et le Japon à l'occasion de ce congé annuel.

Les expatriés japonais travaillant en Algérie y viennent généralement non accompagnés de leur famille, car ils vivent souvent dans des logements provisoires sur des chantiers situés hors des zones urbaines. Les interprètes suivent la même règle, bien que les familles soient parfois tolérées quand le lieu de travail se situe en ville (il faut dire que 50 à $60 \%$ des interprètes sont célibataires, et leurs conditions de vie n'y sont peut-être pas étrangères). De même que les expatriés japonais, les interprètes sont logés gratuitement, mais doivent parfois payer les repas, qui sont généralement préparés à la japonaise par des cuisiniers japonais. Ils bénéficient par ailleurs de l'ensemble des installations de sports et de loisirs mis à la disposition des expatriés.

En fin de contrat, la plupart des interprètes rentrent au Japon et $\mathbf{y}$ attendent la prochaine offre. Certains s'inscrivent à des cours de français pour entretenir leurs connaissances linguistiques. D'autres approfondissent ou élargissent leurs connaissances dans un domaine technique qu'ils choisissent. Puis ils repartent pour un nouveau contrat, et peuvent ainsi faire la navette entre le Japon et l'Algérie pendant plusieurs années de suite.

Les interprètes japonais d'Afrique du Nord ont-ils le sentiment d'être des " professionnels »? Au début du " boom " du marché algérien, ils étaient presque tous amateurs ou débutants. Actuellement, ils peuvent être considérés comme des "semiprofessionnels ". En effet, nombreux sont ceux qui ont plus de cinq ans d'expérience et qui assurent une prestation correcte. D'un autre côté, beaucoup d'entre eux pensent changer d'activité, et rares sont ceux qui se réunissent pour parler de questions professionnelles. Des rumeurs ont bien circulé concernant l'éventuelle création d'une association des interprètes japonais d'Afrique du Nord, mais celle-ci ne s'est jamais matérialisée. Dans l'ensemble, on peut donc dire que les interprètes japonais d'Afrique du Nord ne se sentent pas "professionnels" et n'éprouvent pas le besoin de le devenir.

Cet état de choses peut s'expliquer partiellement par l'attitude des expatriés japonais, qui ne comprennent pas la nature du travail d'interprétation et qui n'accordent pas beaucoup d'estime aux interprètes. Ces derniers sont plutôt considérés comme un "outil de communication " dont le sort est d'ailleurs enviable : ils touchent des salaires relativement élevés et disposent de loisirs assez importants par rapport aux expatriés, puisqu'ils sont libres une fois leur tâche d'interprétation remplie.

Les interprètes étant dans l'ensemble jeunes, on ne peut indiquer de moment précis où ils décident d'interrompre leur carrière d'interprète itinérant. Toutefois, nombreux sont ceux qui se reconvertissent en cours de route. Certains sont embauchés définitivement par les entreprises pour lesquelles ils ont travaillé, d'autres par les agences, d'autres encore peuvent viser les agences de voyage, les entreprises d'import-export, voire la création de leur propre société de traduction. Dans tous les cas, les débouchés sont très limités pour les interprètes japonais-français au Japon et dans des pays nonmaghrébins.

Payés par l'agence qui les envoie, les interprètes sont rémunérés en fonction de leur expérience et du lieu de travail : les salaires sont moins élevés en Tunisie qu'en Algérie, et moins élevés dans les zones urbaines que dans les régions désertiques. Leur revenu annuel se situe aux environs de six millions de yen, auxquels s'ajoute une indem- 
nité en devise locale, qui est actuellement de l'ordre de cinq cents à mille dinars par mois (12 500 à 25000 yen) en Algérie.

Le marché maghrébin, et surtout l'Algérie, fournit actuellement du travail à 250 à 300 interprètes japonais, dont une dizaine de femmes. Continuera-t-il à le faire à l'avenir ? À l'évidence, la réponse est éminemment tributaire de la situation politique dans ces pays d'Afrique du Nord dont la stabilité est relative, ainsi que de la conjoncture économique qui n'est guère prometteuse. Il faut dire aussi que les difficiles conditions de travail, provisoires pour les expatriés mais permanentes pour les interprètes, le manque de contact avec les autochtones, l'impossibilité de mener une vie de famille normale, enfin l'incertitude qui pèse sur les interprètes, qui se retrouvent à la case « départ »à l'expiration de leur contrat, ne les poussent pas à l'optimisme. Ainsi voit-on décroître le nombre des très jeunes interprètes arrivant sur le marché algérien, alors que les interprètes âgés de plus de 40 sont rares. La "bande passante " démographique se resserre et signale le processus de disparition de l'espèce "interprètes japonais en Afrique du Nord".

Toutefois, il est indéniable que ces interprètes ont joué un rôle significatif dans le développement des pays maghrébins et dans le développement de l'économie japonaise, en même temps qu'ils ont aidé à mieux faire connaître les Japonais dans les pays du Maghreb et par là ont eu un rôle très positif.

Je remercie mes collègues interprètes de leurs précieux renseignements qui m'ont beaucoup aidé dans la rédaction du présent article. 